\title{
Hydatid cyst of the omentum
}

\author{
Mahteme Bekele Muleta ${ }^{*}, 1$, Tena Mamo Gamtessa ${ }^{1}$, Mesfin Asefa ${ }^{2}$ \\ ${ }^{1}$ Department of Surgery, St. Paul's Hospital Millennium Medical College, Addis Ababa, Ethiopia, \\ ${ }^{2}$ Department of Pathology, St. Paul's Hospital Millennium Medical College, Addis Ababa, Ethiopia
}

\begin{abstract}
Hydatid cyst is one form of parasitic infestation caused by the larval stage of a cestode species called Echinococcus. The omentum is one of the rarest sites for harboring hydatid cyst. We are reporting a hydatid cyst of the omentum in a 14 year-old Ethiopian patient. Due to its rareness, information about the appearances of hydatid cysts within the omentum is inadequate. We believe that this case report will help to augment the knowledge about hydatid cyst of the omentum by describing and discussing clinical and radiological findings.

Keywords: hydatid cyst, omentum
\end{abstract}

\section{Introduction}

Hydatid Cyst is one form of parasitic infestation caused by the larval stage of a cestode species called Echinococcus. The two main types of the disease are caused by $E$. granulosus and E. multilocularis. Dogs are said to be the definitive host while human and some animals like sheep and cattle are intermediate hosts $[1,2]$. Consumption of unwashed contaminated vegetable and contact with infected animals or contaminated soils is transmission routes for humans, who are an inadvertent intermediate host [3].

In human, the liver is the most common organ being affected by hydatid cyst accounting for $50-75 \%$, followed by the lung $25 \%$ and other organs are rarely affected accounting for $5-10 \%$. It can be manifested as single or multiple cystic lesions in the different organs it affects [1]. Omentum is among the organs which are rarely affected by

Received: September 2016; Accepted after review: December 2016; Published: December 2016.

${ }^{*}$ Corresponding author: Mahteme Bekele Muleta, Assoc. Prof., MD, Department of Surgery, St. Paul's Hospital Millennium Medical College, Gulele subcity, 1271 Swaziland St, Addis Ababa, Ethiopia.

Tel: (251) 923794505

E-mail: mahtemebekele@gmail.com
Hydatid disease, which pose a diagnostic challenge [4]. We are presenting a hydatid disease of the omentum with clinical presentation, imaging, intraoperative, pathologic findings and review of literature.

\section{Case report}

A 14 year-old Ethiopian female patient presented with dull aching lower abdominal pain of one year duration, followed by abdominal swelling of 5 months duration. The swelling was noticed just below the umbilicus, which gradually increased in size to attain the current size. On inspection of the abdomen there was a visible mass below the umbilicus which moved with respiration. On palpation the mass was well defined measuring around 8 $\mathrm{cm}$ in diameter, hard in consistency and moved in all directions. Otherwise, there was no organomegally or shifting dullness.

Laboratory investigation revealed WBC of $9600 / \mathrm{L}$ and hemoglobin level of $14.7 \mathrm{mg} / \mathrm{dl}$. The abdominal ultrasound finding was an anechoic mass in the peritoneum measuring $9.5 \times 6.4 \mathrm{~cm}$; with no connection to the solid organs; suggesting a differential diagnosis of mesenteric cyst or an enteric duplication cyst (Figure 1). 
The CT scan revealed a thick walled cystic mass in the region of ileocecal mesentery measuring $6.7 \mathrm{~cm}$ in diameter having no internal septations or mural calcifications. The bowel appears normal with no wall thickening and the solid organs are normal. The CT suggested a diagnosis of enteric duplication cyst with differential diagnosis of Giant Mickele's diverticulum (Figure 2).

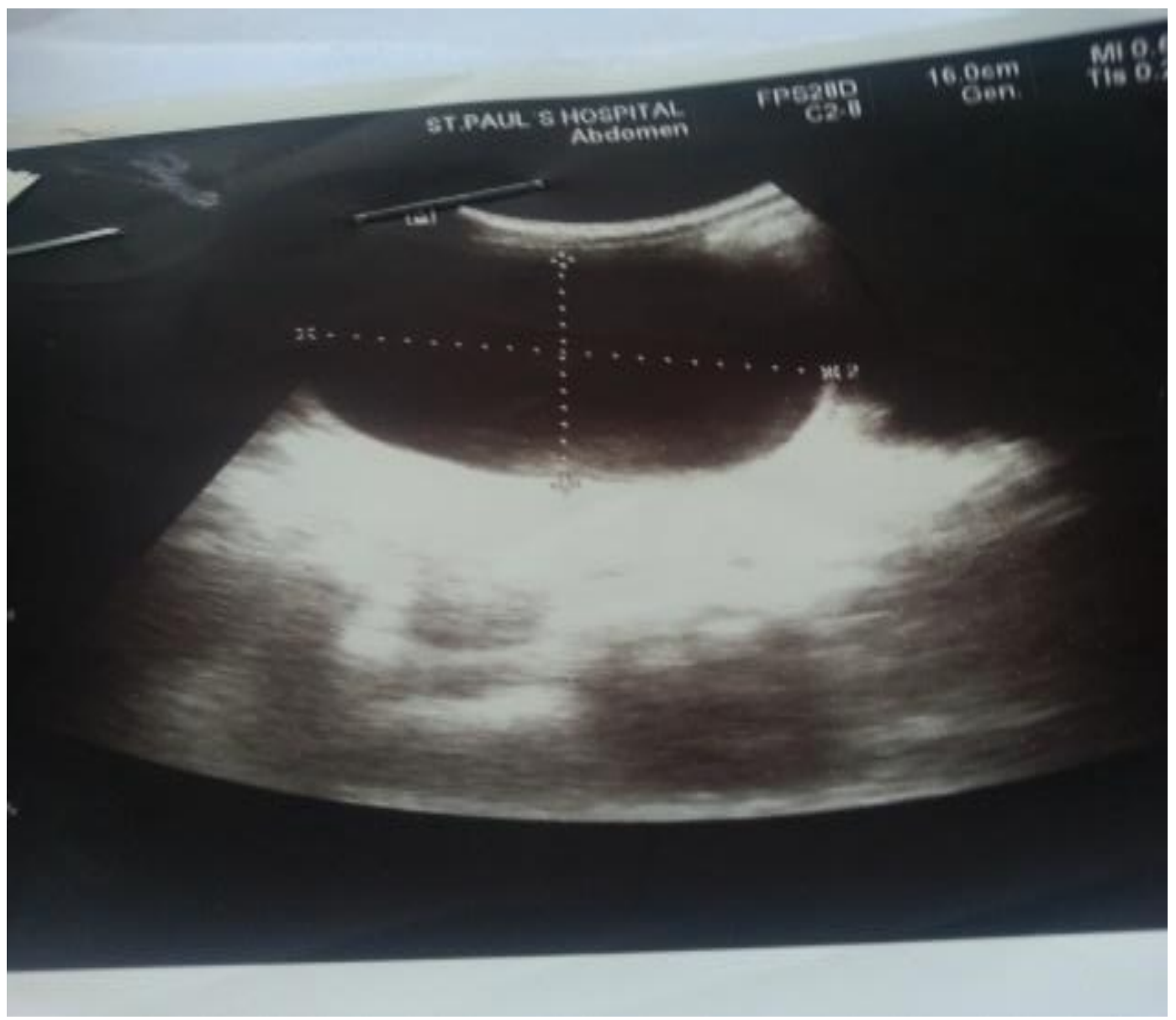

Fig. 1. US showing an anechoic mass in the peritoneum measuring $9.5 \times 6.4 \mathrm{~cm}$

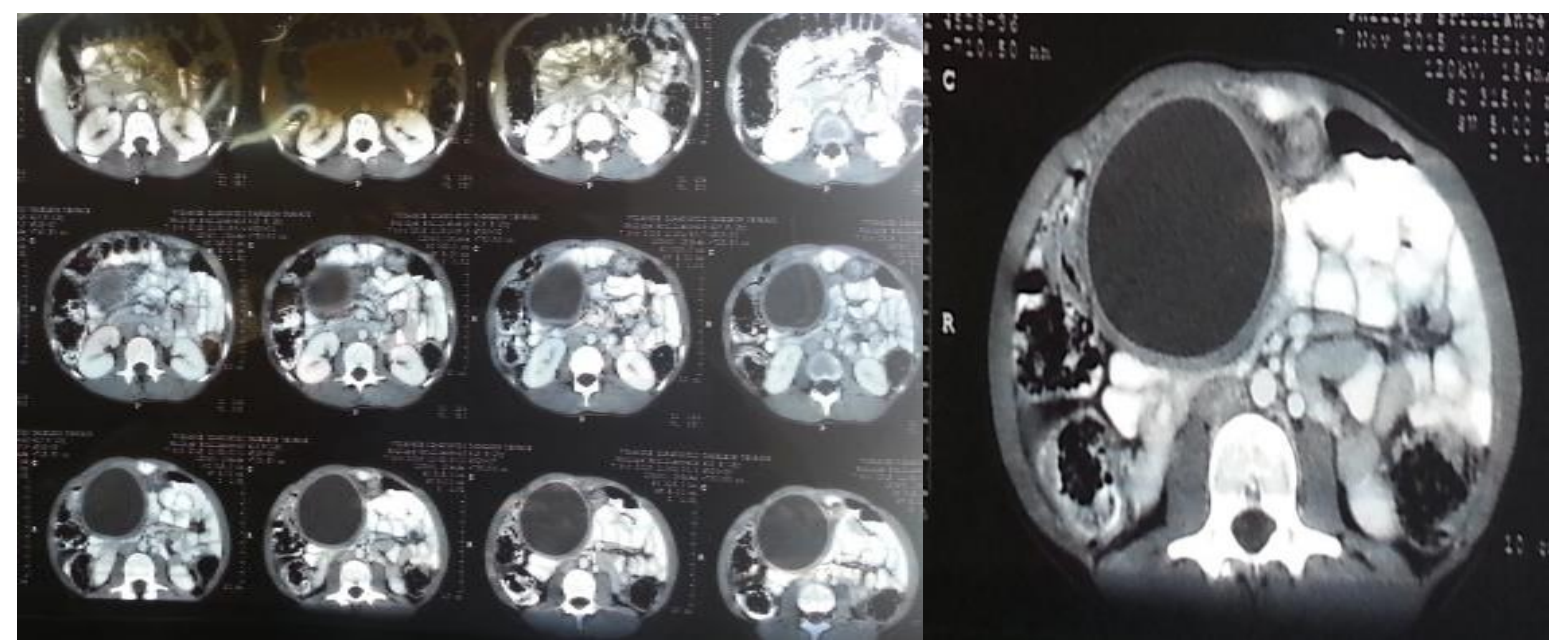

Fig. 2. CT scan: thick walled cystic mass measuring $6.7 \mathrm{~cm}$ in diameter with no internal septations or mural calcification 
With the above diagnosis, the patient was admitted and with sub-umbilical midline incision the abdomen explored. The intraoperative finding was $10 \mathrm{~cm}$ in diameter Cystic mass arising from the omentum for which omental cystectomy was done and sent for histopathologic examination (Figure 3). The patient had uneventful hospital stay and discharged improved in the $4^{\text {th }}$ post-operative day.
The histopathology examination confirmed the diagnosis of hydatid cyst with gross description of a $10 \times 9 \times 6 \mathrm{~cm}$ gray-white nodular cystic mass with attached fat tissue and partly covered by thick mucoid material, and a cut section through the cyst revealed a unilocular cyst filled with serous fluid and lumen covered with thick membraneous mucoid material associated with surface granularities and wall thickness up to $0.3 \mathrm{~cm}$.

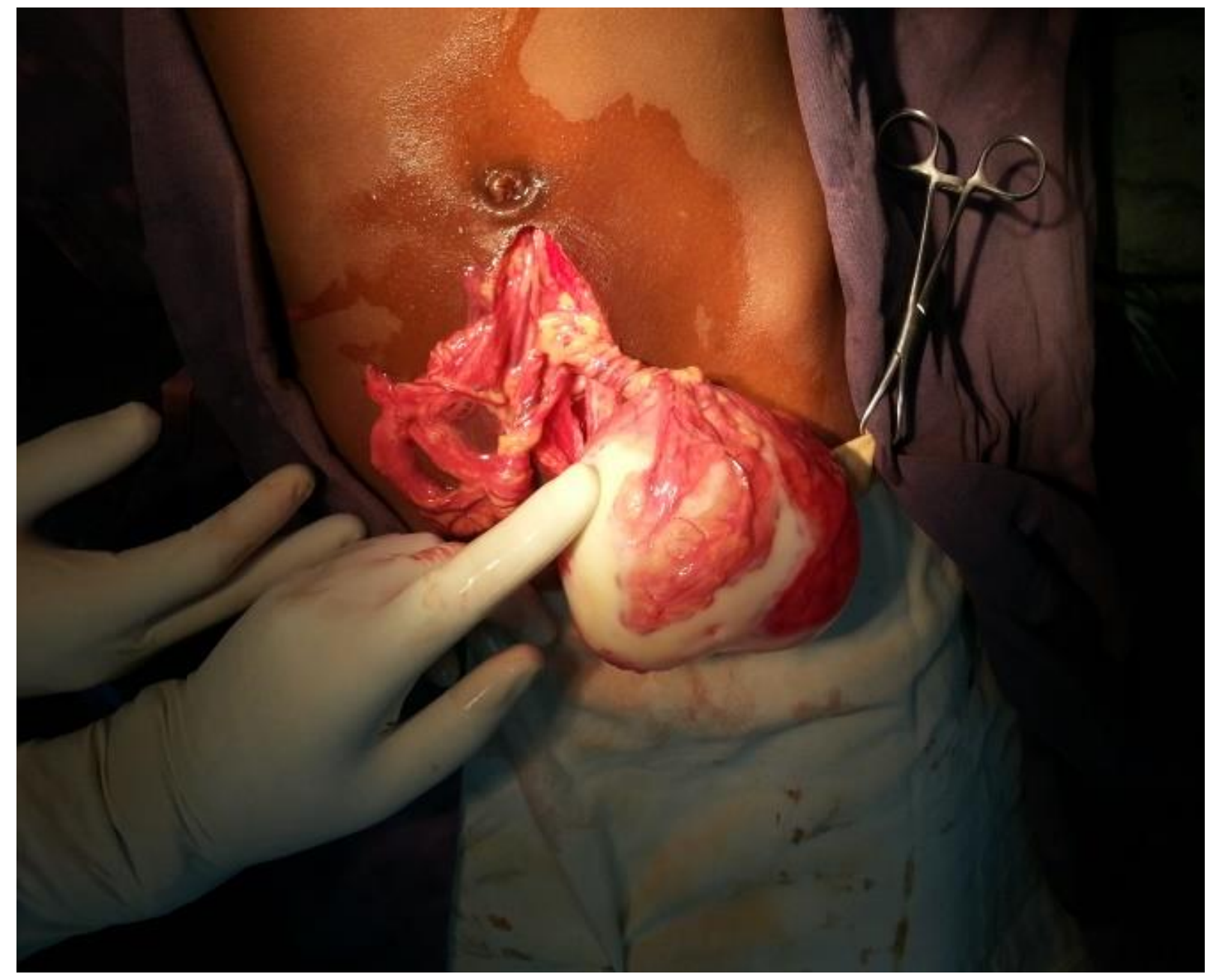

Fig. 3. Gross appearance of the omental cystic nodule, partly surface covered with thick mucoid material

Microscopic examination revealed a fibrous wall from the luminal side lined by hyaline lamellae with dispersed scoleces projecting into the lumen and minimal multifocal lymphocytic and giant cells reactions. Parasitic germ layers are also identified embedded in the fat tissue (Figure 4). 

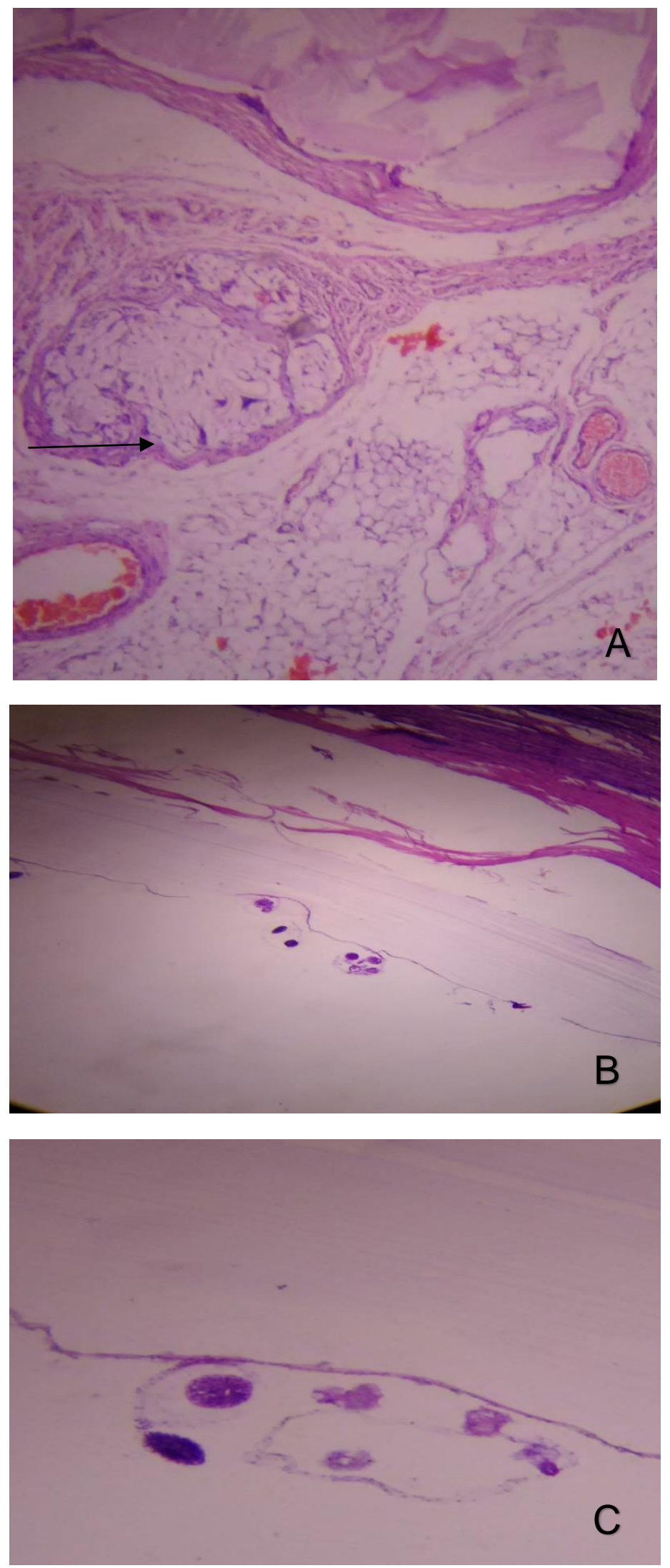

Fig. 4. Pathological examination: A. Parasitic cyst wall and adjacent fat tissue with embedded parasitic germ layers (Arrow head) (HE, x100); B. Hyaline lamellae with focal scolex representing the membraneous mucoid material on gross description (ectocyst, endocyst and pericyst) (HE, x200); C. Parasitic scolex (HE, x400). 


\section{Discussion}

Echinococcal disease is a significant public health problem in South America, the Middle East and Eastern Mediterranean, China, some African countries, and the former USSR $[5,6]$. The estimated number of human infections reaches 1 million worldwide [7]. Although most regions of Africa are poorly researched and limited information is available, several species have been found in the African countries. The most common strain is the G1, highly diffused in the North and East African sheep raising areas [8]. In Ethiopia few researches have been conducted about the disease. The report showed an annual incidence of 2.3 cases per 100000 per year in Bahir Dar and prevalence rate of $1.6 \%$ and $0.5 \%$ in southern part of the country [9].

Clinical manifestation of hydatid cyst depends on the location and the size. Patients can be asymptomatic as cyst grows slowly. Clinical symptoms may include nonspecific abdominal pain, low grade fever, jaundice, urticaria and abdominal mass [10], which is true also in our patient who has presented with abdominal pain and swelling.

The cyst contains three layers which are called the pericyst, ectocyst and endocyst [1]. Different imaging can be used in defining the disease in different sites. Michael et al described a sensitivity of $93 \%$ and $100 \%$ for $\mathrm{U} / \mathrm{S}$ and CT scan respectively [10].

The radiologic finding differs according to the type of the cyst. The early lesions or type I

\section{References}

1. Zinner MJ, Ashley SW. Maingot's abdominal operations, $11^{\text {th }}$ edition. New York City: McGraw-Hill Companies, Inc., 2007.

2. Pedrosa I, Saíz A, Arrazola J, Ferreirós J, Pedrosa CS. Hydatid disease: radiologic and pathologic features and complications. Radiographics 2000; 20(3):795-817.

3. Sayek I, Tirnaksiz MB, Dogan R. Cystic hydatid disease: current trends in diagnosis and management. Surg Today 2004; 34(12):987996.

4. Canda MS, Guray M, Canda T, Astarcioglu H. The pathology of echinococcosis and the current echinococcosis problem in Western hydatid cysts have a non-specific anechoic cystic appearance on ultrasonography (US) and a well-defined, round or oval cystic mass on CT scan. The type II cyst is characterized by the appearance of many daughter cysts and/or matrix developed within the parent cyst with or without wall calcification. Daughter cysts have a lower attenuation value than that of mother cysts on CT scans. Type III cysts on $\mathrm{U} / \mathrm{S}$ appear as cyst calcification as hyperechoic areas with a strong posterior shadow. Cyst calcification can be demonstrated as round, hyper-attenuating areas on CT [11]. Both the U/S and CT scan description of our patient fits to the type I cyst.

The omentum is one of the rare sites for harboring hydatid cyst. In a pathologic report of 80 cases in Turkey only one case was found to be originating from the omentum; Murvet et al couldn't find an image of omental hydatid cyst in his pectoral essay $[4,11]$.

\section{Conclusion}

In our case, both the U/S and the CT scan reading didn't consider a differential diagnosis of hydatid cyst despite the typical description of the image findings. This case report will definitely give a lesson, to radiologist and clinicians, to consider hydatid cyst as a differential diagnosis for such kind of clinical and imaging findings; and care should be taken in handling the cyst to avoid intraoperative rupture.
Turkey (a report of pathological features of 80 cases). Turk J Med Sci 2003; 33:369-374.

5. Jenkins DJ, Romig $T$, Thompson RC. Emergence/re-Emergence of Echinococcus spp.--a global update. Int $J$ Parasitol 2005; 35(11-12):1205-1219.

6. Romig T, Dinkel A, Mackenstedt U. The present situation of echinococcosis in Europe. Parasitol Int 2006; 55:187-191.

7. Fdaladdin YAJ, Alsaggaf AI, Wakid $\mathrm{MH}$. Comparative epidemiological studies on Echinococcosis of local and imported livestock in Al-madina Al-munawwarah in Saudi Arabia. EJHM 2013; 50:108-126. 
8. Grosso G, Gruttadauria S, Biondi A, Marventano S, Mistretta A. Worldwide epidemiology of liver hydatidosis including the Mediterranean area. World J Gastroenterol 2012; 18(13):1425-1437.

9. Kebede N, Mitiku A, Tilahun G. Retrospective survey of human hydatidosis in Bahir Dar, north-western Ethiopia. East Mediterr Health $J$ 2010; 16:937-941.
10. Safioleas M, Misiakos E, Manti C, Katsikas D, Skalkeas G. Diagnostic evaluation and surgical management of hydatid disease of the liver. World J Surg 1994; 18:859-865.

11. Yuksel M, Demirpolat G, Sever A, Bakaris S, Bulbuloglu E, Elmas N. Hydatid disease involving some rare locations in the body: a pictorial essay. Korean J Radiol 2007; 8:531540. 